\title{
EXPERIMENTAL STUDY OF THE ORIENTATION OF MAGNETIC FIELDS IN THE CORONA
}

\author{
P. CHAR VIN \\ Observatoire de Paris, Meudon, France
}

\begin{abstract}
We present polarization measurements obtained in 1970 in the green coronal line with a new coronameter located at the Pic du Midi. The analysis of these data has been conducted with the theory given by the writer in 1964 and 1965. It appears that magnetic field orientations in the Corona can be deduced from the above measurements. First results showing large scale magnetic structures are presented.
\end{abstract}

\section{Introduction}

The writer theoretically studied in 1963 and 1964 the polarization of coronal forbidden lines, and showed that the azimuth of the magnetic field could be determined from the polarization measurements of certain lines, particularly the 5303 green line (Charvin, 1964a, b, 1965).

Since then, several eclipse experiments provided experimental results. The most important contribution has been obtained by Hyder et al. (1968). They have shown:

(1) the validity of the general predictions of the theory (6374 line not polarized; increasing polarization of the 5303 line with the distance from the limb);

(2) moreover, that the polarization of the 5303 line generally lies within numerical values calculated in several simple models;

(3) finally, that the direction of the major electric vector is not radial everywhere, as can be expected from the theory if the magnetic coronal fields are not also radial everywhere.

Unfortunately, their data were not accurate enough to allow the determination of magnetic field orientations.

Preliminary measurements made at Meudon in 1963 (Charvin, 1965) had shown that the sensitivity of the monochromatic coronameter (Charvin, 1963) should be great enough to allow the measurement of the coronal green line polarization without eclipse. Therefore, we rebuilt this instrument to get improved facilities. It is now located at the Pic du Midi Observatory.

This new coronameter is equipped with a $16 \mathrm{~cm}$ diameter coronagraph lens. Specially designed for polarization measurements, it works on-axis, on a field less than $1 \mathrm{~min}$ of arc. Instrumental polarization can be neglected. The disturbing effects due to the sky background and to the K-Corona are compensated with an accuracy which can reach $10^{-4}$. In good observing conditions, this instrument can detect the 5303 green line up to an altitude higher than $1 R_{\odot}$. Its sensitivity is about $0,01 \times 10^{-6}$ $B_{\odot}(1 \AA)$. The following work has been done with this instrument during the last 1970 summer. 


\section{Measurements}

The Corona is photoelectrically observed, point by point, with a 51 or $29 \mathrm{~s}$ of arc aperture. The green line intensity, its polarization and the angle between the major electric vector component of the light and a fixed direction are simultaneously measured. In general, each measurement lasts between 2 and $4 \mathrm{~min}$.

The degree of observed polarization varies within a wide range. As a general rule, it increases with the distance from the limb, and it is higher above polar regions (weak electronic density) than above active regions. The minimum and maximum values measured during these first observations were respectively less than $0,4 \%$ (near the limb), and close to $30 \%$ (at $1 R_{\odot}$ ).

Generally, the percent polarizations measured near the solar limb are about 3 or 4 times weaker than the maximum values that were computed in several models with radial magnetic field (Charvin, 1965). On the contrary, far from the limb, the observed values are frequently similar to the computed ones.

In addition to the depolarizing effects due to the inclination of the magnetic field, the theory provides different possible explanations for these results: for instance, use of underestimated collisional cross-sections; inhomogeneities in the Corona (for example, filamentary structure); overlapping of several magnetic structures along the line of sight.

Indeed, the first magnetic effects play a quite important role, since one often observes important departures from radial configurations. Even, in some cases, one finds the major electric vector parallel to the solar limb. Then, one obtains higher values for the degree of polarization when, using the magnetic maps finally obtained, one takes in account the angular effects. Thus, the agreement with the theoretical computed values frequently becomes much better.

Figure 1 gives an example of the polarization directions observed on July 26, 1970, in the vicinity of Northern solar Pole. During this four-hour observation, the sky brightness generally stayed between 25 and $50 \times 10^{-6} B_{\odot}$. The accuracy of the angular measurements ranges from about $1^{\circ}$ in best cases, to about $12^{\circ}$ for weak polarizations observed under poor conditions (high and variable sky brightness).

One can distinguish regions where the directions of polarization are homogeneous, and regions where the polarization azimuth changes much from point to point. In these apparently inhomogeneous regions, the observed degree of polarization is likely to be frequently diminished by unresolved angular effects in the observed element (here, $51 \mathrm{~s}$ of arc).

\section{Obtaining the Magnetic Field Orientations}

\section{A. PROBLEMS}

The above mentioned theory connects in a simple way the polarization of the light emitted by a small volume element to the magnetic field orientation inside this element. Polarization does not depend on the magnetic field intensity; its direction is only 
dependent on the angular parameters involved in this problem (Charvin, 1964b, 1965). As a final result, and given the values of these angles, the direction of the major electric vector gives, either the azimuth of the magnetic field (i.e. the direction of the magnetic field as projected on the plane normal to the line of sight), or the perpendicular direction. This alternative is governed by strict rules; in many cases, it can be solved in

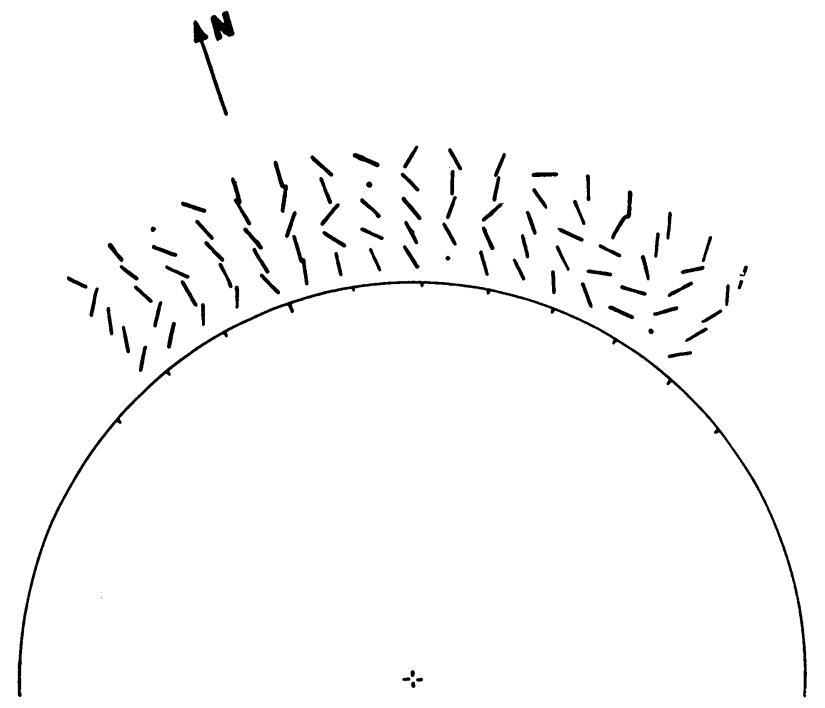

Fig. 1. Observation of the azimuth of the green coronal line between 1 and $5 \mathrm{~min}$ of arc as measured during July 26, 1970 (from 6.50 UT to 10.45 UT; aperture field: $51 \mathrm{~s}$ of arc). The direction of bars gives the direction of the major electric vector component of the light. The dots indicate points where polarization has not been observable.

a fully objective way. However, there are some cases when the choice of the right determination raises a problem. This last ambiguity may be solved by means of internal consistency considerations. This is the first problem.

Let us go now through the second one. Considering the characteristics of the measuring system on one hand (diameter of the observed field, spacing of the observed points, minimum duration necessary to establish the map of a significant part of the Corona), the line of sight effects on the other hand, the existence of large scale magnetic structures in the major part of the Corona seems to be necessary. It has to be that way, so that, more particularly:

(1) the percent polarization has a measurable value on most of the observed elements;

(2) the resulting data have a clear physical meaning. In this respect, the maximum consistency is obtained when polarization has constant magnitude and orientation along the line of sight (Charvin, 1965);

(3) the polarization observed on near elements are (except on both sides of boundary lines) truly connected to the same general magnetic field. Then, the ambiguities previously mentioned can be correctly removed. 
In fact, this assumption that large scale structures do exist in the major part of the Corona, seems to be generally well-founded. For instance, it is well known that structures visible on K-Corona and monochromatic photographs may be, without contradiction, explained as resulting from magnetic structures, and that they seem generally to possess the expected characteristics, i.e. scale and stability. Furthermore, we must emphasize the fact that our polarization data frequently had significant values, and that in addition, equal polarization directions were observed in several cases during 2 or 3 successive days in the same coronal regions. These last points give an experimental support to the existence of such structures. Nevertheless, in some parts of the Corona, the danger still exists that measurements concerning, in fact, 2 or 3 different structures could be improperly ascribed to the same magnetic pattern. This risk could be greatly reduced by increasing the density of the measured points.

\section{B. OBTAINING MAGNETIC MAPS}

The achievement of the right determination of magnetic orientations (magnetic field azimuth parallel or normal to the measured direction), and the drawing of the maps are then performed both together. However, for better comprehension, we present Figure 2 as well as Figure 3.

In order to check our results, up to a certain extent, we proceeded as follows:

First, we outlined a map of the field orientation, taking only in account the values of the measured angles and the general properties of magnetic fields (continuity, conservation). This leads to the identification of most of the main structures. At the end of this step, the arches 1, 2, 4 and 5, the polar features 3 and the regions 6 and 8 are, in the example given in Figure 3, clearly distinguished.

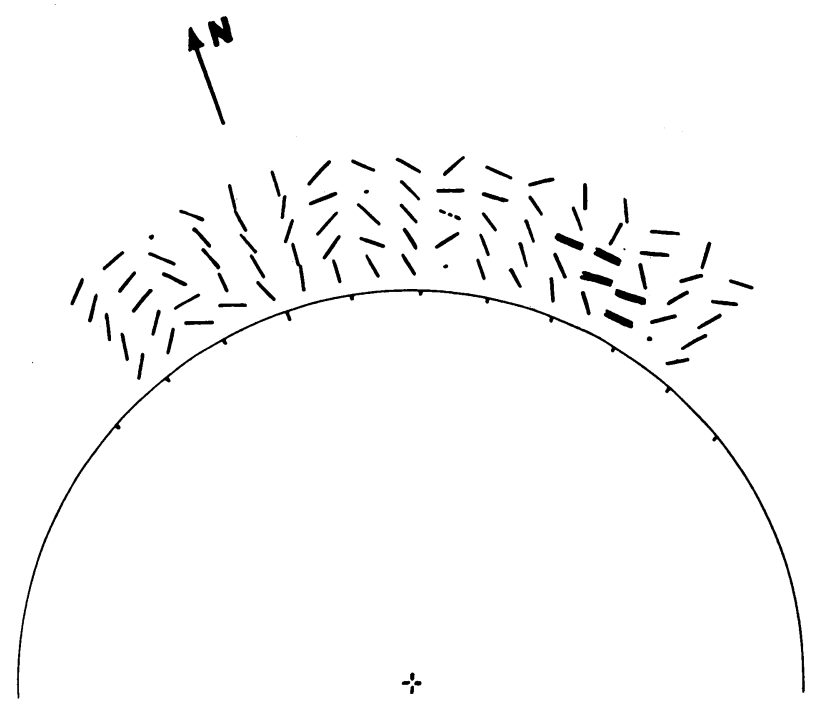

Fig. 2. Diagram of magnetic field orientations as projected on the plane normal to line of sight. The double bars indicate points where the magnetic field has a strong component along the line of sight. 


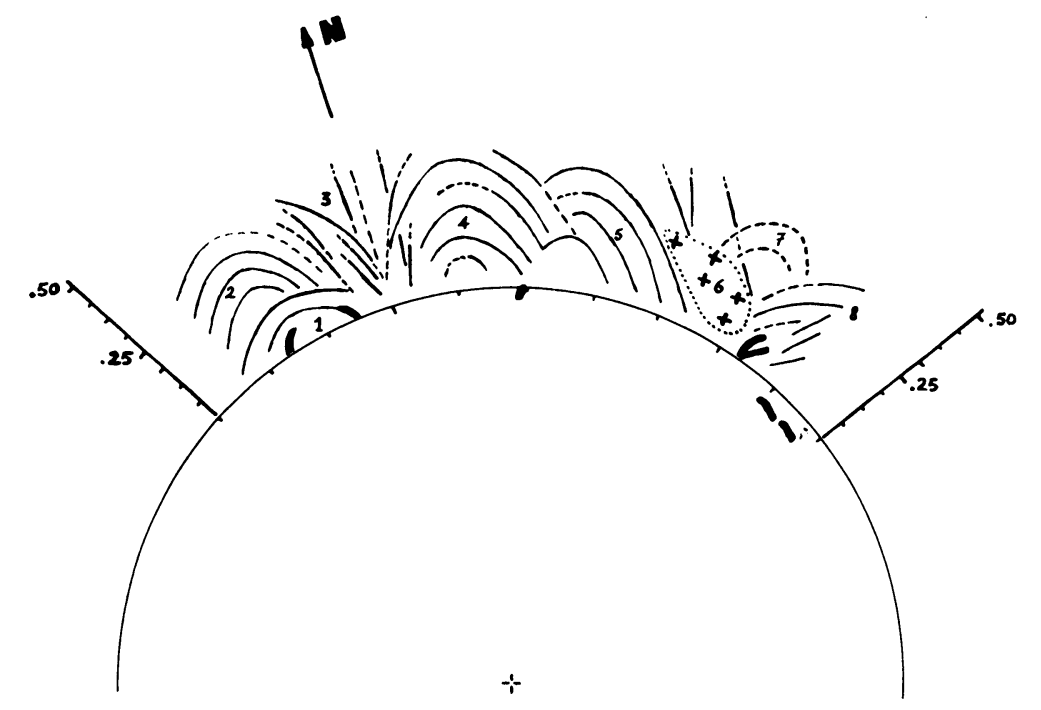

Fig. 3. Schematic map of the coronal magnetic field (July 26, 1970). The prominences and filaments observed on spectroheliograms are shown on the map.

Second, in order to check and to refine these preliminary results, we made use of the degrees of polarization and of the isophotes. Thus, we are able to fix in a better way the apparent boundaries of the different structures. In difficult cases, the close connections often existing between the green line intensities and the magnetic structures make some extrapolations possible. Thus, the existence of structure 7 is suggested by the isophotes.

An ultimate analysis could be undertaken if the electronic densities were known with precision. It would consist of the computation of models concerning the studied regions, and of their comparison to the observations. This last step would, particularly make possible the estimation of the inclination of the structures with the line of sight.

\section{FIRST RESULTS}

When considering Figure $3^{*}$, one first notices a similarity between such a map and the appearance of numerous eclipse photographs as well as monochromatic ones. Differences should also exist, at least those resulting from some properties of our instrument (weak space resolution, linear response), and from the different ways in which the polarizations of monochromatic emissions on one hand, and the white light or monochromatic intensities on the other hand, should be weighted along the line of sight. Let us mention that this similarity is also visible on the other maps we drew above active regions (see the footnote).

More precisely, Figure 3 shows:

- polar features 3 which rise from a very restricted area;

* Several other maps have been presented at this Symposium. They will be published later. 
- arch structures 1, 2 and 4 which connect the Northern Pole to lower latitude regions;

- feature 6, whose main characteristic is a strong magnetic component along the line of sight;

- the relations between the quiescent prominences, the filaments and the coronal magnetic structures. These relations, also clearly visible on the other maps, indicate that the magnetic field must be parallel to the apparent axis of the prominences, i.e. to the long axis of the filaments. This seems to give a new support to the KippenhahnSchlüter theory (1967).

A detailed study of the connection between coronal matter and magnetic structures will be published later.

\section{Conclusions}

The experimental study of the coronal magnetic field orientations by means of forbidden lines polarization provides a new method in solar research. In fact, the observations which have been presented here are the first accurate observations of these phenomena made without eclipse. They are also the first ones which allow, through specific optical measurements, the drawing of magnetic maps in the Corona. Hence, it is easy to realize that the right use of such observations still requires additional work. Otherwise, our main purpose, when making these first observations, was primarily to test the method and the reducing procedures. At this point, a comparison of our results with those provided by other methods seems to be important. In this respect, a comparison with pictures taken in the green line, and also with structures computed with the method developed by Newkirk and Altschuler (1969) still remains to be done.

However, the following points should be already noticed:

(1) The large scale magnetic structure of the Corona has been observed during the course of these first observations. The actual measurements of significant degrees of polarization, often close (after correction) to theoretical values, provide first evidence of this. These measurements also afford a good estimate of the extent and stability of these structures. The existence of several structures visible at the same apparent place during 2 or 3 successive days, produce one more proof. The identification of some peculiar features, as the diverging appearance of the polar magnetic field, or the relations between the coronal magnetic field and prominences, seems to be a third remarkable point.

These results substantiate the general acceptance that structures visible on eclipses and monochromatic pictures correspond to lines of force of the coronal magnetic field.

(2) The good internal consistency of the results here obtained gives a second verification of the theory, more achieved than the verification obtained by Hyder et al. (1968).

(3) We think the magnetic field maps are already obtained in a rather satisfying way, even if some parts or details have not yet the required degree of certainty. The main difficulties we met do not arise from the $90^{\circ}$-ambiguity existing for certain angular values, but much more from a too weak density of the observed elements (compared 
to the extent and complexity of actual coronal structures). We think a systematic use of a lower field aperture (about $30 \mathrm{~s}$ of arc), along with a doubling of the observed point density would strongly reduce these difficulties. Particularly, we think that the degree of subjectivity yet existing in the interpretation of data could be practically nullified.

Some improvements of coronameter should allow this doubling without a correlative increase of the total observing time.

(4) We think these first results should stimulate further eclipse observations of coronal line polarization. The method we are using has in fact two limitations: its weak space resolution, and its long scanning time. It does not seem possible, even in the best case, to measure in a whole day more than 350 or 400 points with this instrument.

(5) Finally, we think this method should yield new valuable information in the field of coronal physics. Besides the study of magnetic structures in themselves and in connection with photospheric fields, their knowledge should allow us to interpret, much better than now, measurements of white light and monochromatic intensities. New evidence concerningin homogeneities in the Corona as well as magnetic field intensities is likely to be obtained.

\section{Acknowledgements}

I thank Prof. J. Rösch, Director of the Pic du Midi Observatory, whose hospitality allowed me to set up the coronameter in the best conditions. I am grateful to $\mathrm{Mr} \mathbf{J}$. Arnaud for invaluable help during the course of observations.

This research is partly supported by the 'Direction des Recherches et Moyens d'Essais'.

\section{References}

Altschuler, M. D. and Newkirk, G., Jr.: 1969, Solar Phys. 9131.

Charvin, P.: 1963, Compt. Rend. Acad. Sci. 256, 368.

Charvin, P.: 1963, Compt. Rend. Acad. Sci. 256, 1078.

Charvin, P.: 1964a, Compt. Rend. Acad. Sci. 258, 1155.

Charvin, P.: 1964b, Compt. Rend. Acad. Sci. 259, 733.

Charvin, P.: 1965, Ann. Astrophys. 28, 877.

Hyder, C. L., Mauter, H. A., and Shutt, R. L.: 1968, Astrophys. J. 154, 1039.

Kippenhahn, R. and Schlüter, A.: 1957, Z. Astrophys. 43, 36.

\section{Discussion}

Severny: How could you discriminate between the polarization due to magnetic field and just due to the scattering effect in the line examined by Lyot (observationally) by Thiessen and others and which is essentially nonmagnetic? Why do you ascribe all the effect to magnetic field?

Charvin: The polarization I am observing is not due to the magnetic field. In fact, this polarization is due to a diffusion process similar to that which, as you point out, has been observed by Lyot in the prominence lines. It should occur even without magnetic fields, because it is due to the anisotropy of the radiation field producing the line. However, the effects of this type are magnetic field dependent. In the present case, the magnetic field acts by reducing the percent polarization and by tilting the polarization plane according to the theoretical rules I have given. I make use of these last effects for the determination of the magnetic field in the corona. 
Beckers: Can you explain why the polarization is parallel or at right angles to the field lines? From the contribution by Dr. House I understand that the angle of polarization depends also on the amount of precessing of the bound electron in the magnetic field.

Charvin: Quantum mechanics gives the answer. As the strength of the magnetic field is certainly higher than $10^{-5}$ or $10^{-6} \mathrm{G}$ in the regions of the corona $\mathrm{I}$ am observing, we have not taken into account precession effects in the case of forbidden coronal lines. In this case, the polarization is either normal to the field or parallel to the field as projected on the plane normal to the line of sight. Let us consider a small volume element in the corona. The angle between the normal to the Sun, which is the symmetry axis of the radiation field, and the magnetic field, which is the quantization axis, gives the sign of the polarization. When this angle increases, the polarization decreases, then cancels for Van Vleck's value (about $55^{\circ}$ ), and finally becomes negative. Roughly speaking, because of line of sight effects, the direction of the field is directly given by the observation for angles observed between $35^{\circ}$ and $55^{\circ}$. For the observed values lower than $35^{\circ}$ the ambiguity is not present because of the magnitude of the percent polarization.

Lamb: It should be borne in mind that in the analysis described by Charvin it is not necessary to consider level crossing-interference, and hence the dependence of the plane of polarization on field strength described by Dr House does not need to be taken into account. 